\title{
Allgemeine Infektionsrisiken und ihr Einfluss auf die Heilung von Knochen und Weichteilen
}

\author{
Philipp Wagner, Carsten Hopf, Karl Heinrich Winker
}

\section{Zusammenfassung}

Die Prävention von Infektionen sollte oberstes Ziel der chirurgischen Therapie sein. Die exakte Evaluierung des Patienten und seine Komorbidität decken eventuell vorhandene allgemeine Risikofaktoren für eine Wundheilungsstörung auf. Nicht selten handelt es sich um multifaktoriell belastete Patienten, deren Risikofaktoren sich gegenseitig potenzieren können. Häufig liegen Erkrankungen der Perfusion vor, wobei zwischen arterieller, venöser und einer gemischten Insuffizienz unterschieden werden muss, um eine korrekte Therapie einleiten zu können. Da der Grundumsatz allein durch das Operationstrauma und die benötigten Bausteine zur Wundheilung auf das Doppelte ansteigen können, sind mangelernährte Patienten besonders gefährdet. Die gesellschaftlich anerkannten Süchte „Nikotin“ und „Ethanol“ haben direkt oder indirekt, wie beispielsweise die Incompliance bei Alkoholikern, erhebliche negative Auswirkungen. Starke Raucher haben eine um das Dreifache erhöhte Kom- plikationsrate bei der Wundheilung, alkoholkranke Patienten zeigen eine bis zu viermal höhere postoperative Infektrate und eine bis um das Zehnfache verlängerte Frakturheilung. Bei einem ungenügend eingestellten Diabetes mellitus findet sich ein bis $\mathrm{zu}$ fünffach gesteigertes Wundheilungsstörungsrisiko. Die immer mehr an Bedeutung zunehmende Gruppe der älteren und alten Patienten zeigt, bedingt durch verminderten Knochenstoffwechsel und der verminderten biologischen Potenz, ebenfalls ein um das Dreifach gesteigertes Wundheilungsrisiko. In diesem Zusammenhang erlangt die suffiziente Therapie der Osteoporose einen erheblichen Stellenwert auch in der Prävention von Refrakturen.

In der heutigen operativen Medizin tritt daher die Indikationsstellung, unter besonderer Berücksichtigung der individuellen Risikoprofile des Patienten, neben der technischen Durchführbarkeit eines Eingriffes immer mehr in der Vordergrund.

\section{Einleitung}

Postoperative Infektionen, und speziell Wundheilungsstörungen nach chirurgischen Eingriffen, stellen für den Patienten eine zusätzliche, erhebliche Belastung dar. Sie sind zudem volkswirtschaftlich durch den protrahierten Heilverlauf außerordentlich bedeutungsvoll.

In diesem Zusammenhang stellt sich immer die Frage, ob es sich um eine kompli-

OP-JOURNAL 2005; 21: 214-219

(c) Georg Thieme Verlag KG Stuttgart · New York zierte Wunde oder einen komplexen Patienten handelt. Bereits Bagatelltraumen können bei disponierender Grunderkrankung zu einer problematischen Wunde führen. Um ein erhöhtes Wundheilungsrisiko im Vorfeld zu erkennen oder eine pathologische Wundheilung postoperativ effektiv behandeln zu können ist es notwendig, die ursächliche Grunderkrankung zu identifizieren, um gezielte Maßnahmen und Therapien ergreifen zu können.

In der heutigen Zeit gerät die Frage nach der technischen Versorgungsmöglichkeit durch immer besser angepasste Verfahren zunehmend in den Hintergrund. Viel- mehr erlangt die Frage nach der grundsätzlichen Operationsindikation im Hinblick auf Grundleiden und Risikofaktoren einen immer größeren Stellenwert.

\section{Physiologische Wundheilung}

Die Beurteilung des Risikos der Wundheilungsstörung setzt zunächst eine Analyse der physiologischen Wundheilung voraus.

Im normalen Wundheilungsprozess werden drei Phasen unterschieden: In der Entzündungsphase wird nach der Gewebetraumatisierung zunächst das Gerinnungssystem aktiviert, Wachstumsfaktoren werden durch Thrombozyten sezerniert und leiten die nachfolgenden Wundheilungsereignisse ein. Proteasen bewirken ein endogenes Wunddebridement, Granulozyten wehren über Phagozytose Mikroorganismen ab, Zytokine und Monozyten stimulieren die Angiogenese und eine Fibroblastenproliferation.

Während der Granulationsphase sprosst mesenchymales Gewebe entlang der provisorischen Matrix in den Defekt ein. Nekrotisches Gewebe wird an der Einwanderungsfront weiter abgebaut und eine neue extrazelluläre Matrix angelagert. Im Verlauf nimmt die Zellzahl massiv zu und die Epidermis wandert über das Granulationsgewebe ein. An der Wanderungsfront beginnen die Keratinozyten zusammen mit den mesenchymalen Zellen des Granulationsgewebes die Basalmembran zu generieren. Hierbei kommt es entscheidend auf die zeitliche und räumliche Balance der einzelnen mediatorischen Faktoren an. Während sich das Granulationsgewebe ohne epithelialen Einfluss ausbildet, benötigen Keratinozyten die Stimulation ihres Wachstums durch das mesenchymale Gewebe.

Nach dem epithelialen Wundverschluss ist die Barrierefunktion der Haut weitest gehend wieder hergestellt. Die epidermale Hyperproliferation der Keratinozyten 
normalisiert sich. Im darunter liegenden Bindegewebe erfolgt der Umbau des zellreichen und matrixarmen Granulationsgewebes in ein zellarmes, matrixreiches Narbengewebe [23].

\section{Pathologische Wundheilung}

Durch die Störung der komplexen physiologischen Wundheilungsmechanismen aufgrund lokaler Störfaktoren oder systemischer Grundleiden resultieren Wundheilungsstörungen auf den unterschiedlichsten Ebenen. Einen Überblick über mögliche Ursachen gibt die Tab.1.

Im Folgenden sollen einige für die chirurgische Therapie wesentlichen und unter dem typischen Patientengut häufigen Erkrankungen genauer betrachtet werden.

Häufig liegen mehrere die Wundheilung störende Faktoren vor. Diese Risikofaktoren können sich gegenseitig beeinflussen und potenzieren.

$\mathrm{Zu}$ beachten ist, dass selten monokausale Zusammenhänge bestehen. Oftmals finden sich multifaktorielle Risikogruppen, wobei sich einzelne Faktoren zusätzlich gegenseitig beeinflussen: Ein adipöser Patient mit übermäßigem Alkoholkonsum und Nikotinabusus wird eventuell auch einen latenten oder manifesten Diabetes mellitus aufweisen und hat offensichtlich ein vielfach höheres Risiko als der alleinige Raucher.

Die folgende Betrachtung der einzelnen Risikofaktoren ist deshalb nur im Zusammenhang sämtlicher patientenspezifischer Leiden zu sehen.

\section{Perfusionsveränderungen:}

Eine gestörte Perfusion ist eine der häufigsten Ursachen für eine schlecht heilende Wunde. Wichtig ist die Differenzierung zwischen arterieller, venöser oder einer gemischt arteriell-venösen Pathologie.

Vor allem in der Granulationsphase kommt es darauf an, dass in der Wunde Grundbausteine in Form von Aminosäuren, Energie und Sauerstoff lokal in ausreichenden Mengen zur Verfügung gestellt werden. Eine Mangelernährung oder Erkrankungen mit kataboler Stoffwechsellage (Tumorkachexie) verringern offensichtlich das Angebot an diesen Bausteinen. Oftmals und häufig auch in Kombination bei den meist älteren und multimorbiden Patienten bestehen Zirkula-
Tab. 1 Erkrankungen mit Prädisposition zur Wundheilungsstörung
Gefäßveränderungen (häufigste Ursache)

- chronisch venöse Insuffizienz

- pAVK

- primäres/sekundäres Lymphödem

Chronisch entzündliche Erkrankungen

- Pyoderma gangraenosum

- Psoriasis

- Vaskulitiden

- perforierende Dermatosen

- rheumatoide Arthritis

Hämatologische Erkrankungen

- Gerinnungsstörungen

- Sichelzellenanämie

- Polycythaemia vera

Verschiedene Leiden

- Bestrahlung

- Pernionen

tionsstörungen, welche den ausreichenden Transport in das Zielgebiet „Wunde“ vermindern. Nicht selten sind diese Veränderungen, arteriell oder venös, sogar ursächlich für das Auftreten von Wunden verantwortlich (Ulcus cruris, diabetisches Fußsyndrom). Die basale Gefäßdiagnostik liefert entscheidende Hinweise, ob es sich um eine rein chronisch venöse Insuffizienz handelt, oder ob eine arterielle Komponente vorliegt. Der „Tibio-brachiale-Index“, also die Bestimmung des arteriellen Druckquotienten, stellt beispielsweise mit einfachen Mitteln ein sinnvolles diagnostisches Instrument dar. Gemessen wird der arterielle Druck an den Arterien im Sprunggelenkbereich mit Blutdruckmanschette und TaschenDoppler. Dieser wird dividiert durch den ebenso gemessenen arteriellen Druck am Oberarm.

Bei rein venösen Störungen gilt heute die suffiziente Kompressionsbehandlung in Kombination mit interaktiven Wundauflagen als Standard in der Therapie. Kommt es zu keiner befriedigenden Besserung sollte die Diagnose jedoch immer kritisch überprüft werden. Vielleicht liegt noch eine weitere, die Abheilung behindernde Grunderkrankung vor?

\section{Ernährung}

Hier leuchtet die Kausalität eines zu geringen Angebotes an Grundbausteinen bei Mangelernährung und dadurch bedingt die erhöhte Inzidenz von Wundheilungsstörungen ein. Zudem ist zu beachten, dass ein chirurgischer Eingriff per se bereits eine Stresssituation mit einer gesteigerten Stoffwechsellage darstellt. Der
- Verbrennung
Metabolische Erkrankungen

- Diabetes mellitus

- Fehlernährung

- Gicht

Infektionen

- Bakterien

- Pilze

- Parasiten

- geschwächte Immunabwehr (z. B. HIV, Cortisontherapie)

Tumoren

- primäre kutane Tumoren

- exulzerierte Metastasen

Ernährung

- Drogenkonsum (Alkohol)

- Kachexie

- Adipositas katabole Stoffwechselzustand einerseits und der erhöhte Bedarf an Substraten für die Wundheilung andererseits kann eine Steigerung des Energieumsatzes von ca. $1 \mathrm{kcal} / \mathrm{h} / \mathrm{kg}$ Körpermasse auf bis zu 2 kcal/h/kg Körpermasse bedingen.

Der chirurgische Eingriff stellt eine Stresssituation mit gesteigertem Stoffwechsel dar. In Kombination mit einer heilenden Wunde kann der Energieumsatz des Organismus bis auf das Doppelte gesteigert sein.

Neben dem Substanzmangel für die Wundheilung muss dem Ernährungszustand des Patienten auch eine wesentliche Rolle bei der Aufrechterhaltung der Infektabwehr zugeschrieben werden.

Bereits Studien vor über 60 Jahren zeigten einen Zusammenhang zwischen perioperativem Gewichtsverlust und operativer Mortalität einerseits sowie Fehlernährung chirurgischer Patienten und Infektionsraten andererseits. Nachgewiesen sind Beeinträchtigungen der spezifischen und nicht-spezifischen Immunabwehr bei Mangelernährung. Sie betrifft sowohl Komponenten der humoralen als auch der zellulären Abwehr. Obwohl die genauen Mechanismen noch unbekannt sind, besteht kein Zweifel, dass eine Störung der immunregulatorischen Funktionen von Zytokinen, inflammatorischen Mediatoren und Stresshormonen resultiert. Ähnliche pathophysiologische Reaktionen findet man auch nach ausgedehnten Gewebetraumatisierungen. Diese Tatsache unterstreicht den Stellenwert eines ausreichenden Ernährungsstatus insbesondere dann, wenn Trauma 
und chirurgischer Stress synergistisch mit einem defizitären Ernährungszustand zu einer katabolen Stoffwechsellage führen.

Das hauptsächliche Problem in der klinischen Praxis besteht in einer objektiven Bewertung des Grades der Unterernährung. Die möglichen biochemischen Verfahren korrelieren nicht hinreichend. Daher basieren viele der heute aktuellen Scoring-Systeme auf einer Kombination mit anamnestisch erhobenen Befunden. Beachtung finden akuter Gewichtsverlust, gastrointestinale Erkrankungen, funktionelle Handikaps des Patienten, Verlust von subkutanem Fettgewebe und Umfang des Muskelschwundes. Die Kombination von Ausprägung des akuten Gewichtsverlustes und Serumalbuminspiegel wird beispielsweise für die Bestimmung des „Nutritional Risk Index“ (NRI) verwendet und erlaubt in gewissem Maße eine objektive quantifizierbare Einschätzung des Ernährungszustandes.

Hauptenergielieferant sind Kohlenhydrate und Fette. Unter physiologischen Bedingungen wird ein Verhältnis von 70\% Glukose zu 30\% Fett empfohlen. Bei einem hypermetabolischen Zustand ist es erforderlich, den Fettanteil auf bis zu $50 \%$ zu erhöhen. Da die Glukoseoxidationsrate begrenzt ist würde ein Überangebot zu Hyperglykämie, osmotischer Diurese, gestörter Fettmobilisation und gesteigerter Kohlendioxidproduktion führen.

Zucker und speziell Glukose dient in der Wundheilung als Substrat für Leukozyten und hat einen proteinsparenden Effekt. Auch Fette als Kalorienträger helfen Protein einzusparen und haben präkursorische Funktionen für Prostaglandine und Leukotriene. Wenn möglich sollte die Verabreichung enteral erfolgen, da hier negative Effekte wie bei parenteraler Gabe riskierte immunsuppressive Wirkungen vermieden werden und zudem eine Störung der physiologischen Barrierefunktion des Darmes präventiert wird. Eine weitere tragende Rolle spielt das Nahrungsprotein: Für die Wundheilungsprozesse werden Aminosäuren als Substrat für die Proteinsynthese bei der Fibroblastenproliferation, der Kollagensynthese, der Neovaskularisierung und der Phagozytosefähigkeit der Leukozyten benötigt. Der Bedarf kann von etwa $1 \mathrm{~g} / \mathrm{Tag} /$ kg Körpergewicht auf das Doppelte ansteigen.
Neben den Hauptnährstoffen besteht bei der Wundheilung auch ein gesteigerter Bedarf an Vitaminen, Mineralien und Spurenelementen: Für Vitamin C sind fördernde Effekte als Kofaktor bei der Kollagen- und Glukosaminoglykansynthese sowie antioxidative Eigenschafen nachgewiesen. Vitamin A ist unerlässlich für die Granulation und Epithelialisierung der Wunde. Positive Wirkungen zeigt auch die Verabreichung von Mineralien. Signifikant nachweisbar ist dieser Effekt allerdings erst bei lang bestehenden, chronischen Wunden [11].

\section{Risikofaktor Nikotin}

Durch den Nikotinkonsum bedingte Effekte auf die Haut begründen sich unter anderem auf einer direkten Vasokonstriktion durch Erhöhung des Sympatikotonus und einer gesteigerten Vasopressinsekretion. In Kombination mit der Zunahme von Carboxyhämoglobin kommt es zu einer vermehrten Gewebeischämie. Eine Erhöhung der Blutviskosität und Veränderung der Gerinnungseigenschaften ist durch eine Steigerung der Thrombozytenaggregation sowie eine Zunahme des Plasmafibrinogens bedingt und verschlechtert die periphere Gewebeversorgung zusätzlich.

Eine Steigerung der Aktivität der Metalloproteinase-1 führt zu einer Degeneration von kollagenen Fasern. Andererseits induziert Rauchen über eine verstärkte Elastaseaktivität den Abbau des Elastans in der Dermis [15]. Weiterhin wird eine direkte Interaktion mit hemmendem Effekt auf immunologischer Ebene diskutiert, indem unter anderem die Differenzierung von Monozyten und die Migration von Leukozyten beeinflusst wird $[20,10]$. Die Knochenheilung betreffend ist experimentell erwiesen, dass Nikotin das Einwachsen von Gefäßen in eine Spongiosaplastik verlangsamt. Bestandteile des Rauches fördern die Entkalkung des Knochens durch eine erzeugte Calcitoninresistenz, erhöhen die Knochenresorption an Frakturenden und stören die Osteoblastenfunktion [13].

Somit verändert das Rauchen sowohl in der frühen als auch späten Wundheilungsphase wesentlich die physiologischen Prozesse.

Verschiedene Studien haben klinisch den Einfluss des Rauchens auf die postoperative Wundheilung untersucht: „Fast jeder dritte starke Raucher muss mit einem postoperativen Infekt rechnen“ [24].
Rauchen von mehr als 20 Zigaretten täglich steigert die Komplikationsrate bei der Wundheilung um das Dreifache und verlängert die Knochenbruchheilung signifikant.

Einflüsse auf die Mikrozirkulation, vor allem bedeutend bei Replantationen, wurden eindeutig bestätigt: Während des Genusses von ein bis zwei Zigaretten konnte bei Untersuchungen mithilfe des Laser-Dopplers eine Abnahme des peripheren Flusses um bis zu 29\% verzeichnet werden. Zudem fand sich (im Gegensatz zu den Nichtrauchern) bei der Gruppe der chronischen Raucher praktisch keine Erholung während der ersten 10 Minuten nach dem Rauchen [13].

Nach Lappen- und Vollhauttransplantationen wurde eine signifikante Beziehung zwischen der Ausprägung des Zigarettenkonsums und dem Auftreten postoperativer Nekrosen aufgezeigt. Der Konsum von mehr als 20 Zigaretten pro Tag steigerte die Komplikationsrate auf das Dreifache, wobei Gelegenheitsraucher und ehemalige Raucher ein vernachlässigbares, nicht signifikant höheres Risiko einer Transplantatnekrose aufwiesen. Mediane Laparotomienarben waren bei Rauchern über das Doppelte breiter als bei Nichtrauchern [15].

Nach isolierten Tibiafrakturen fanden sich bis zur klinischen Heilung bei Rauchern deutlich verlängerte Zeiträume (im Mittel 166 vs. 134 Tage), während gleichzeitig eine höhere Rate verzögerter Frakturheilungen auftrat. Es bestand eine direkte Proportionalität bezüglich der Zeit bis zur klinischen Heilung, der Anzahl des täglichen Zigarettenkonsums, dem Patientenalter und dem Weichteilschaden. Auch bei der radiologischen Beurteilung bis zum Heilungsabschluss von geschlossenen oder erstgradig offenen Frakturen fanden sich bei Rauchern deutlich längere Zeiten (244 vs. 144 Tage).

In Tierversuchen konnte gezeigt werden, dass nachteilige Effekte des Rauchens auf die Revaskularisierung innerhalb von zwei Wochen reversibel waren, wobei sich die Viskosität des Blutes bereits nach zwei Tagen besserte [13].

Alle Studien stimmen darin überein, dass Rauchen als eindeutiger Risikofaktor für eine gestörte Knochen- und Wundheilung zu identifizieren ist. 
Als Schlussfolgerung ist zu empfehlen, dass sowohl prä- als auch postoperativ das Rauchen zumindest für eine gewissen Zeitraum einzustellen ist. Man muss dabei bedenken, dass der Rauchstopp nicht nur langzeitig, sondern schon nach recht kurzer Zeit positive Auswirkungen zeigt. Die Angaben über die Dauer schwanken in der Literatur jedoch: Es finden sich Empfehlungen von bis zu 3 Wochen präoperativ und bis zu 4 Wochen Abstinenz nach dem Eingriff, beziehungsweise bis zum Abschluss der Rehabilitation, um Komplikationen zu minimieren.

\section{Konsum von Ethanol}

„Das Risiko, eine postoperative bakterielle Infektion zu erleiden, war für Patienten mit einem Alkoholgenuss von mehr als $60 \mathrm{~g} /$ Tag viermal höher als für das Restkollektiv“" [24].

Der schädliche Einfluss von Alkohol auf Pankreas, Leber, ZNS und Schleimhäute ist unumstritten. Die möglichen Gründe der verzögerten Fraktur- und Wundheilung bei chronisch alkoholkranken Menschen sind multifaktoriell: Neben der im Tierexperiment nachgewiesenen direkt hemmenden Wirkung von Alkohol auf die Kallusbildung und den negativen Einfluss auf die Wundheilung, bedingt der chronische Alkoholkonsum eine ganze Reihe von Stoffwechseldefiziten, welche sekundär zu einer schlechter heilenden Wunde führen: Eine Ethanoldiät führt zu einer Mangelernährung, einer verminderten Resorption von Mineralien, Proteinen und Vitamin D. Es resultiert eine hyperparathyroidotische Mineralstoffwechsellage, die Proteinsynthese der Leber ist reduziert, Polyneuropathien und trophische Störungen sind keine Seltenheit. Eine direkte Immunsystemdepression scheint die hohe postoperative Infektrate weiter zu begünstigen.

Nicht vernachlässigen darf man sicherlich die Indolenz und Disziplinlosigkeit bei Alkoholikern, welche durch verfrühte Fehlbelastung eine hohe Pseudarthrosenund Refrakturrate fördern.

Studienergebnisse untermauern diese Überlegungen: Bei Oberschenkelfrakturen fanden sich Heilungszeiten von 55,9 vs. 18,7 Wochen bei nicht chronischen Alkoholkonsumenten. Die Infektraten waren bei Alkoholikern fast viermal so hoch (16,6 vs. 4,3\%), Pseudoarthrosen und Refrakturen fanden sich doppelt so häufig und eine verzögerte Frakturheilung wurde mit 25 vs. 2,2\% beobachtet.
Insgesamt spricht diese Studie von 50\% Komplikationen aller Art bei alkoholkranken Menschen im Vergleich zu 12\% des Restkollektivs.

Die gleichen Autoren untersuchten auch Unterschenkelfrakturen: Hier war bei Alkoholikern die Heilungsdauer fast zweifach verlängert, die Infektionsrate war ebenfalls viermal so hoch, Pseudarthrosen und Refrakturen traten viermal häufiger auf und eine mangelnde Kallusbildung als Ausdruck einer verzögerten Frakturheilung war mehr als doppelt so häufig zu finden. Die Gesamtkomplikationsrate betrug 50 vs. 17\%. In diesen Studien erlitt also jeder zweite alkoholkranke Patient eine Komplikation nach Frakturversorgung [2].

Übermäßiger Alkoholkonsum hat einen direkten Einfluss auf die Wundheilung mit einer bis zu vier mal höheren Infektrate und verlängert die Knochenbruchheilung bis zum Zehnfachen.

\section{Alter}

Die erhöhte Lebenserwartung und die Überalterung der Bevölkerung bedingen einen dramatischen Anstieg der „Alterschirurgie“. Zurzeit erleiden in Deutschland jährlich ca. 60000 Menschen eine Schenkelhalsfraktur (90/100000 Einwohner ) wobei Hochrechnungen zufolge diese Zahl bis zum Jahr 2010 auf 135/ 100000 Einwohner steigen soll [4]. Chirurgie und Unfallchirurgie im Alter ist aufgrund der Zunahme multimorbider Patienten über 70 Jahre zu einer immensen interdisziplinären Herausforderung geworden, um das Ziel einer frühestmöglichen komplikationsarmen Wiederherstellung von Form und Funktion sowie die Wiedereingliederung in die soziale Umwelt des Verletzten zu erreichen. Die vielen Zusatzerkrankungen und die altersbedingte Degeneration aller Organe, auch der Haut, sowie des Muskel- und Skelettsystems beeinflussen die Heilung nachhaltig.

Im Alter laufen regenerative und reparative Prozesse langsamer ab. Dies hat auch Einfluss auf die komplizierten Prozesse der Wundheilung in Ihren 3 Stadien.

So muss bei über 60-Jährigen mit einem dreimal höheren Wundheilungsrisiko gerechnet werden. Dabei werden sämtliche Stadien der Wundheilung erfasst:

- Senkung der Zellproliferation

- Reduktion der Kapillarneubildung

- Verlangsamung der Epithelisation
- Reduktion der Keratonizytenproliferation in Abhängigkeit mitogener Reize

- Verminderung der Wundkontraktion [12].

Deshalb ist es wichtig mit einen minimalen Operationstrauma nach optimaler interdisziplinärer Vorbereitung eine frühe Belastungsstabilität zu erreichen.

Eine Wundinfektion im hohen Alter führt zu einer signifikanten Erhöhung der Mortalität. Auch der Knochenstoffwechsel nimmt im Alter ab mit verlangsamten regenerativen und reparativen Prozessen. Die Mitbehandlung der Osteoporose und auch deren Prävention muss Kernaufgabe des Chirurgen sein.

Durch Behandlung der diagnostizierten Osteoporose wird eine Zweitfraktur in bis zu $60 \%$ der Patienten vermieden.

Über 60-Jährige haben ein dreifach höheres Wundheilungsrisiko und durch ihren verminderten Knochenstoffwechsel sowie aufgrund der verminderten biologischen Potenz eine verlangsamte Heilungstendenz der Knochen.

Durch Behandlung der Osteoporose wird das Risiko einer Zweitfraktur um nahezu $60 \%$ gesenkt. Prävention ist die Hauptaufgabe aller Ärzte in der Zukunft, um das Gesundheitswesen bezahlbar zu halten.

\section{Glucocortikoide}

Verschiedene Medikamente wie Cortison und viele nichtsteroidale Antirheumatika haben einen hemmenden Einfluss auf die Frakturheilung [3]. Glukokortikoide können die Transskription von RNS in DNS in Abhängigkeit von der Dosis sowohl hemmen als auch stimulieren und üben so Kontrolle über Syntheseprozesse bestimmter Enzyme aus. Somit haben sie auch direkten Einfluss auf die komplexe Wundheilung [19].

Im Rahmen der Wundheilung sind Glucocorticoide für die verlangsamte Proliferation von Epidermis-, Entzündungs- und Fettgewebszellen verantwortlich. Die Synthese von kollagenem Bindegewebe durch Fibroplasten und die Freisetzung von allergiespezifischen Mediatoren aus Mastzellen sowie die Pigmentbildung von Melanozyten wird beeinflusst. Die Bedeutung der Glucocortikoide für das Auftreten von Wundinfektionen und Wundheilungsstörungen ist aber unbestritten. Es ist nachgewiesen, dass Steroide einen antagonistischen Effekt auf Wachstumsfaktoren und Kollagensynthese bei der Wundheilung ausüben. 
Der negative Einfluss von Steroiden auf die Wund- und Knochenheilung ist dosisabhängig.

\section{Chemotherapeutika}

Chemotherapeutika und Zytostatika üben einen negativen Einfluss auf die zelluläre Proliferation von Mesenchymzellen und inflammatorischen Zellen aus. Durch die myelotoxische Wirkung der Chemotherapeutika wird die Produktion von für die Wundheilung bedeutenden Wachstumsfaktoren und Zytokinen negativ beeinträchtigt. Experimentell konnte gezeigt werden, dass bestimmte Chemotherapeutika zu den potentesten Inhibitoren der Wundheilung zählen [22]. Klinische Ergebnisse am Menschen konnten diese Ergebnisse nicht bestätigen. Allerdings zeigten rekonstruktive Eingriffe an Füßen bei Rheumapatienten unter chemotherapeutischer Medikation eine um $32 \%$ erhöhte Infektionsrate [6].

Chemotherapeutika zählen zu den potentesten Inhibitoren der Wundheilung.

\section{Diabetes mellitus}

Diese Systemerkrankung begünstigt Wundheilungsstörungen durch die begleitende Mikroangiopathie und Neuropathie sowie die Koexistenz weiterer Risikofaktoren. Ein signifikanter Einfluss auf die Knochenheilung konnte in der Literatur nicht gefunden werden.

Die klinischen Untersuchungsergebnisse zur Bedeutung des Diabetes mellitus als Risikofaktor einer Wundheilungsstörung bei primär heilenden Wunden sind widersprüchlich. Während einige Studien keinen Effekt beschreiben, sehen andere das Risiko von Diabetikern, eine postoperative Wundheilungsstörung zu bekommen, fünffach höher als bei der Normalpopulation [21].

Beim Diabetiker ist durch Beeinträchtigung des Vitamin-C-Transports die Kollagensynthese reduziert [9].

Der nicht kontrollierte bzw. nicht oder schlecht eingestellte Diabetes mellitus ist prädisponierend für die gestörte Wundheilung durch die gestörte Leukozytenfunktion. Vor allem Chemotaxis und Phagozytose laufen vermindert und weniger zuverlässig ab. Tierexperimentell konnte durch frühzeitige Insulingabe diese Beeinträchtigung teilweise oder ganz ausgeglichen werden [1]
Prinzipiell wird die gestörte Wundheilung beim Diabetiker von vielen Faktoren beeinflusst. Die Sensibilitätsstörungen, bedingt durch die Neuropathie, begünstigen rezidivierende Traumen. Die mikrovaskulären Duchblutungsstörungen führen zu einer verminderten Gewebeperfusion. Die gestörte zelluläre Immunabwehr führt wiederum häufig zu Wundinfektionen.

Diabetes mellitus mindert auch die Knochenqualität und beeinflusst die Knochenheilung negativ. Dies konnte in tierexperimentellen Studien nachgewiesen werden. Diese Störung konnte durch Behebung des Insulinmangels und gute Glukoseeinstellung behoben bzw. geheilt werden [7].

In der Literatur ist bisher der negative Einfluss von Diabetes mellitus auf den Heilungsverlauf von Frakturen nicht wissenschaftlich belegt worden. Boddenberg [7] konnte in seiner Literaturrecherche über die Heilungsdauer von Knöchelfrakturen bei Diabetikern und in eigenen Studien keinen signifikanten Unterschied zu Nichtdiabetikern feststellen.

In einer Studie wurde nachgewiesen, dass $25 \%$ der Patienten mit Osteomyelitis als Grunderkrankung einen Diabetes mellitus haben.

Laboruntersuchungen konnten beweisen, dass mikrovaskuläre Alterationen mit dem Blutzuckerspiegel korrelieren. Unkontrollierte Diabetiker haben eine schlechtere Wundheilung als gut eingestellte Diabetiker mit einer erhöhten Infektionsrate von 72 gegenüber 55\% und damit verbundenen längeren Verweildauer von 24 Tagen gegenüber 10 Tagen [18].

Die Rate von Wund- und Knochenheilungsstörungen bei Diabetikern ist abhängig von der perioperativen optimalen Einstellung des Blutzuckerspiegels. Schlecht oder ungenügend eingestellte Diabetiker haben ein bis zu fünf mal höheres Wundheilungsstörungsrisiko.

\section{Psoriasis}

Die Psoriasis vulgaris (Schuppenflechte) bedingt im Allgemeinen eine erhöhte Prädisoposition für einen postoperativen Infekt: Die meisten Studien geben eine erhöhte Infektionsrate von 5,5 bis $17 \%$ nach Implantation einer Hüft- oder Knie-TEP an.
Eine Studie über verschiedene chirurgische Eingriffe, wobei orthopädische Eingriffe einbezogen wurden, fand keine erhöhte Infektionsrate [16]. Auch Beyer et al. [5] fanden bei ihren Nachuntersuchungen von 34 Patienten mit Psoriasis nach Implantation einer Knie-TEP kein erhöhtes Wundheilungsrisiko bei entsprechender perioperativen Antibiotikaprophylaxe. Christophers betrachtet die Psoriasis als Modell für die epitheliale Abwehr. Für die Erforschung von Mechanismen der epithelialen Abwehr am Menschen diente die psoriatisch erkrankte Haut als ein Fundus für viele neue Erkenntnisse. Die psoriatischen Schuppen bieten ausreichend Substanzen für die antimikrobielle Abwehr [8]. Zusammenfassend kann man sagen, dass bei entsprechender sorgfältiger OP-Vorbereitung und perioperativer Antibiotikaprophylaxe kein signifikantes erhöhtes Infektionsrisiko besteht und die Wundheilung nicht beeinträchtigt wird.

Ob Psoriasis ein prädisponierender Faktor für eine Wundheilungsstörung ist, hängt von der perioperativen Infektionsprophylaxe ab.

\section{Tumoren}

In der vorliegenden Literatur ist kein signifikanter Einfluss von Tumoren auf die Wundheilung zu finden, wenn man Knochentumoren und -metastasen mit ihrem bekannt negativen Einfluss auf die Heilung pathologischer Frakturen herausnimmt.

Allerdings zeigt die Wundheilung bei Tumorkranken im Verlauf eine Abweichung in Form einer verzögerten Heilung aufgrund der Hypoproteinämie, VitaminC-Mangel und sekundärer Anämie.

In der Literatur konnte aber ein Zusammenhang zwischen einer präoperativen Hypoproteinämie oder einer präoperativen Corticosteroideinnahme auf die Wundinfektionsrate nachgewiesen werden [17].

Aus diesen Erkenntnissen kann ein direkter Einfluss von Tumoren auf die Wundheilung nicht hergestellt werden.

Tumoren haben wegen ihrer katabolen Auswirkung auf den Stoffwechsel einen indirekten negativen Einfluss auf die Wundheilung. 


\section{Adipositas}

Bei Adipösen besteht ebenfalls ein erhöhtes Risiko, eine postoperative Wundkomplikation zu erleiden. Dies erlangt eine immense Bedeutung, wenn man bedenkt, dass nach einer im Jahr 2003 vom Statistischen Bundesamt durchgeführten Studie in Deutschland rund $49 \%$ der erwachsenen Bevölkerung über 18 Jahren an Übergewicht leiden. Es konnte nachgewiesen werden, dass Wundkomplikationen nach Implantation einer KnieTEP bei Adipösen zu 22\% aufgetreten sind gegenüber $2 \%$ in der Kontrollgruppe. Das heißt, es ist bei Adipösen mit einem zehnfach höheren Risiko zu rechnen, eine Wundheilungsstörung $\mathrm{zu}$ bekommen [25]. Außerdem kommen noch erschwerend das größere OP-Trauma mit größerer Wundlänge, stärkerem Hakenzug und verlängerte OP-Dauer hinzu. Diese Kombination führt zu einer verzögerten oder gestörten Wundheilung [14].

Postoperative Wundkomplikationen können bei Adipösen in Abhängigkeit vom BMI bis zu 10-mal häufiger auftreten als bei Normalgewichtigen

\section{Radiatio}

Ionisierende Strahlung (Alpha-, Beta- und Gammastrahlung) kann die biologische Zellfunktion, insbesondere die Proteinsynthese, stören. Es lassen sich 4 Stadien der Schädigung unterscheiden:

- Früherythem

- Dermatitis erythematodes

- Dermatitis bullosa

- Dermatitis gangraenosa

Die Wundheilungsstörungen im bestrahlten Gewebe sind multifaktoriell und werden hauptsächlich durch Gewebehypoxie und Organellenbeschädigung verursacht.

Wundheilungsstörungen im bestrahlten Gewebe sind multifaktoriell.

\section{Literatur}

${ }^{1}$ Andreassen TT, Oxlund $\mathrm{H}$. The influence of experimental diabetes and insulin treatment on the biochemical properties of rate skin incisional wounds. Acta Chir Scand 1987; 153: 405

${ }^{2}$ Bartsch H, Jahr F. Komplikationen und Ursachen der verzögerten Knochenbruchheilung des chronischen Alkoholikers. Orthop Praxis 1985; $1: 64-68$

${ }^{3}$ Beck A, Krischak G, Sorg T. Influence of diclofenac (group of nonsteroidal anti-inflammatory drugs) on fracture healing. Arch Orthop Trauma Surg 2003; 123 (7): 327-332

${ }^{4}$ Beck A, Rüter A. Therapiekonzepte bei Schenkelhalsfrakturen. Chirurg 71; $240-$ 248 (3): $347-354$

${ }^{5}$ Beyer CA, Hanssen AD, Lewallen DG, Pittelkow MR. The Journal of bone and joint surgery, British volume, 1991; 73: 258-259

${ }^{6}$ Bibbo C, Anderson RB, Davis WH, Norton J. The influence of rheumatoid chemotherapy, age, and presence of rheumatoid nodules on postoperative complications in rheumatoid foot and ankle surgery: analysis of 725 procedures in 104 patients. Foot and Ankle Int 2003; 24(2): 118

${ }^{7}$ Boddenberg U. Heilungsdauer von Fuß- und Knöchelfrakturen bei Diabetikern: Literaturübersicht und Darstellung eigener Fälle. Zentralbl Chir 2004; 129: 453-459

${ }^{8}$ Christophers E. Psoriasis - epitheliale Abwehr und die Entwicklung des metabolischen (Insulin-Resistenz-) Syndroms. Akt Dermatol 2004; 30: 289-292

${ }^{9}$ Goodson WH, Hunt TK. Studies on wound healing in experimental diabetes mellitus. J Surg Res 1977; 22: 221 - 227

${ }^{10}$ Guinet E, Yoshida K, Nouro-Shirazi M. Nicotinic environment affects the differentiation and functional maturation of monocytes derived dendritic cells. Immunology Letters 2004; 95: 45-55

${ }^{11}$ Halangk W, Weiß G, Tautenhahn J, Lippert H. Influence of Nutritional Status on Wound Healing and Wound Infection. Viszeralchir 2004; 39: 177-180

${ }^{12}$ Holt DR, Kirk SJ, Regan MC. Effect of age on wound healing in healthy human beings. Surgery 1992; 112: 293-298

${ }^{13}$ Hoogendoorn JM, Simmermacher RKJ, Schellekens PPA, van der Werken C. Rauchen ist nachteilig für die Heilung von Knochen und Weichteilen. Unfallchirurg 2002; 105: $76-81$

14 Klima St. Adipositas als Risikofaktor Z Orthop Ihre Grenzgeb 2004; 142: 505-507

${ }^{15}$ Krug M, Wünsche A, Blum A. Addiction to tobacco and the consequences for the skin. Hautarzt 2004; 55: $301-316$
${ }^{16}$ Lambert JR, Wright V. Surgery in patients with psoriasis and arthritis. Rheumatol Rehabil 1979; 18: $35-37$

${ }^{17}$ Mc Phee IB, Williams RP, Swanson CE. Factors influencing wound healing after surgery for metastatic disease of the spine. Spine 1998 Mar 15; 23(6): 726-732

${ }^{18}$ McCambell B, Wasif N, Rabbitts A, StaianoCoico L, Yurt RW, Schwartz S. Diabetes and burns: retrospective cohort study. J Burn Care Rehabil 2002 May-Jun; 23(3): 157-66

${ }^{19}$ McCoy BJ, Diegelmann RF, Cohen IK. In vitro inhibition of cell growth, collagen synthesis and prolylhydroxylase activity by triamcinolone acetonide. Pro Soc Exp Biol Med 1980; 163: $216-222$

${ }^{20}$ Razani-Boroujerdi S, Singh SP, Knall C, Hahn FF, Pena-Philippides J-C, Kalra R, Langley RJ, Sopori ML. Chronic nicotine inhibits inflammation and promotes influenza infektion. Cellular Immunology 2004; 230: 1 -9

${ }^{21}$ Scheithauer M, Riechelmann H. Übersicht Teil II: Die gestörte kutane Wundheilung. Laryngo-Rhino-Otol 2003; 82: 36-39

${ }^{22}$ Shamberger RC, Deveraux DF, Brennan MF. The effects of chemotherapeutic agents on wound healing. Int Adv Surg Oncol 1981; 4: $15-58$

${ }^{23}$ Smola H, Eming S, Hess S, Werner S, Krieg T. Wundheilung und Wundheilungsstörungen: Moderne Konzepte zur Pathophysiologie und Therapie. Dt Ärztebl 1998; 43: A2802/B-2387/C-2235

${ }^{24}$ Stopinski J, Staib I, Weissenbach M. Haben Nikotin- und Alkoholmissbrauch Einfluss auf das Auftreten postoperativer bakterieller Infektionen? Langenbecks Arch Chir 1993; 378: 125-128

${ }^{25}$ Winiarsky R, Barth P, Lotke P. Total knee arthroplasy in morbidly obese patients. J Bone Joint Surg Am 1998 Dec; (12): 1770-1774

\section{Dr. med. Philipp Wagner Assistenzarzt Dr. med. Carsten Hopf Oberarzt \\ Univ.- Prof. Dr. med. Karl Heinrich Winker \\ Chefarzt}

Klinik für Unfall-, Hand- und Wiederherstellungschirurgie HELIOS-Klinikum

Nordhäuser Strasse 74

D-99089 Erfurt 